\title{
Spin polarizations and spin Hall currents in a two-dimensional electron gas with magnetic impurities
}

\author{
C. Gorini, P. Schwab, and M. Dzierzawa \\ Institut für Physik, Universität Augsburg, 86135 Augsburg, Germany \\ R. Raimondi \\ CNISM and Dipartimento di Fisica "E. Amaldi," Università Roma Tre, 00146 Roma, Italy
}

(Received 16 May 2008; published 29 September 2008)

\begin{abstract}
We consider a two-dimensional electron gas in the presence of Rashba spin-orbit coupling, and study the effects of magnetic $s$-wave and long-range nonmagnetic impurities on the spin-charge dynamics of the system. We focus on voltage induced spin polarizations and their relation to spin Hall currents. Our results are obtained using the quasiclassical Green function technique, and hold in the full range of the disorder parameter $\alpha p_{F} \tau$.
\end{abstract}

DOI: 10.1103/PhysRevB.78.125327

PACS number(s): $72.25 .-\mathrm{b}$

In the field of spintronics, much attention has recently been paid to spin-orbit related phenomena in semiconductors. One such phenomenon is the spin Hall effect, i.e., a spin current flowing perpendicular to an applied electric field. ${ }^{1-4}$ It is now well known that, for linear-in-momentum spin-orbit couplings such as the Rashba or Dresselhaus ones, the spin Hall current vanishes exactly in the bulk of a disordered two-dimensional electron gas (2DEG) ${ }^{5-8}$ This can be understood by looking at the peculiar form of the continuity equations for the spin, as derived from its equations of motion in operator form. $^{9-11}$ For a magnetically disordered 2DEG things are, however, different, and a nonvanishing spin Hall conductivity is found. ${ }^{12-14}$ Once more, a look at the continuity equations provides a clear and simple explanation of the effect: ${ }^{13}$ a term, whose appearance is due to magnetic impurities, directly relates in-plane spin polarizations, induced by the electric field, to spin currents. As the former, which have been the object of both theoretical and experimental studies, ${ }^{15-20}$ are influenced by the type of nonmagnetic scatterers considered, we forgo the simplified assumption that these are $s$ wave and take into account the full angle dependence of the scattering potential. Besides going beyond what is currently found in the literature, where, in the presence of magnetic impurities, the nonmagnetic disorder is either neglected or purely $s$ wave, our approach also shows the interplay between polarizations and spin currents in a 2 DEG. ${ }^{21}$ We note that in the correct limits our results agree with what is found in Ref. 14. On the other hand a discrepancy with Ref. 12 arises.

For the calculations we rely on the Eilenberger equation for the quasiclassical Green function in the presence of spinorbit coupling. ${ }^{22}$ The spin-orbit energy is taken to be small compared to the Fermi energy, i.e., $\alpha p_{F} \ll \epsilon_{F}$-or equivalently $\alpha \ll v_{F}$, and the standard metallic regime condition $1 / \tau \ll \epsilon_{F}$ is also assumed. Here $\alpha$ is the spin-orbit coupling constant, $p_{F}\left(v_{F}\right)$ the Fermi momentum (velocity) in the absence of such coupling, and $\tau$ the elastic quasiparticle lifetime due to nonmagnetic scatterers. Our results hold for a wide range of values of the dimensionless parameter $\alpha p_{F} \tau$ since this is not restricted by the above assumptions. Contributions of order $\left(\alpha / v_{F}\right)^{2}$ are neglected throughout. We focus on intrinsic effects in the Rashba model; extrinsic ones, ${ }^{23}$
Dresselhaus terms, ${ }^{24}$ and hole gases ${ }^{25}$ are not taken into account. Finally, weak localization corrections, which could in principle play an important role, ${ }^{11}$ are beyond the scope of our present work.

The Hamiltonian of the 2DEG, confined to the $x-y$ plane, reads

$$
H=\frac{\mathbf{p}^{2}}{2 m}-\mathbf{b} \cdot \boldsymbol{\sigma}+V(\mathbf{x}),
$$

with $\mathbf{b}=\alpha \mathbf{e}_{z} \times \mathbf{p}$ the Rashba internal field, $\boldsymbol{\sigma}$ the vector of Pauli matrices, and $V(\mathbf{x})=V_{\mathrm{nm}}(\mathbf{x})+V_{\mathrm{m}}(\mathbf{x})$ the disorder potential due to randomly distributed impurities. ${ }^{26}$ Nonmagnetic scatterers give rise to $V_{\mathrm{nm}}(\mathbf{x})$,

$$
V_{\mathrm{nm}}(\mathbf{x})=\sum_{i} U\left(\mathbf{x}-\mathbf{R}_{i}\right)
$$

while $V_{\mathrm{m}}(\mathbf{x})$ describes magnetic $s$-wave disorder

$$
V_{\mathrm{m}}(\mathbf{x})=\sum_{i} \mathbf{B} \cdot \boldsymbol{\sigma} \delta\left(\mathbf{x}-\mathbf{R}_{i}\right) .
$$

Both potentials are treated in the Born approximation, and the standard averaging technique is applied.

To begin with, we look at the continuity equation for the $s_{y}$ spin polarization, ${ }^{13,27}$

$$
\partial_{t} s_{y}+\partial_{\mathbf{x}} \cdot \mathbf{j}_{s_{y}}=-2 m \alpha j_{s_{z}}^{y}-\frac{4}{3 \tau_{s f}} s_{y},
$$

where the second term on the right-hand side is due to magnetic impurities. Here $\tau_{s f}$ is the spin-flip time that stems from the potential (3) [cf. Eq. (13)]. Under stationary and uniform conditions the above equation implies a vanishing spin current-hence a vanishing spin Hall conductivity-unless magnetic disorder is also present, in which case instead

$$
j_{s_{z}}^{y}=-\frac{2}{3 m \alpha \tau_{s f}} s_{y} .
$$

Since the out-of-plane polarized spin current is related to the in-plane spin polarization, we now use simple physical arguments to explain how the latter is generated by an applied voltage. ${ }^{15,28}$ Since the Fermi surface is shifted by an amount 

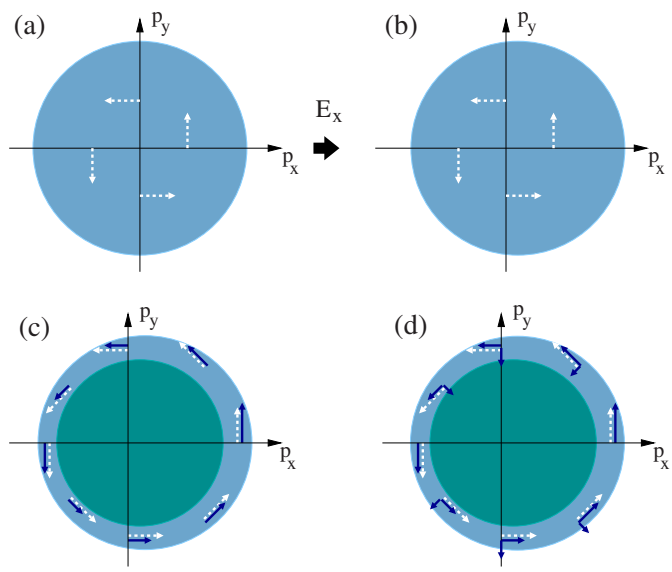

FIG. 1. (Color online) [(a) and (b)] The Fermi-surface shift, $\delta p=|e| \mathcal{E} \tau$, due to an applied electric field along the $x$ direction. The white arrows show the direction of the internal field $\mathbf{b}$. [(c) and (d)] Shifted bands and spin polarization in stationary conditions. (c) Asymmetric shift of the two bands when angle-dependent scattering is present. The long dark (blue) arrows show the contributions to the spin polarization arising from a sector $\mathrm{d} \varphi$ of phase space. (d) When magnetic disorder is turned on, additional contributions orthogonal to the internal field $\mathbf{b}$ appear, here shown by the short inward and outward pointing (blue) arrows. Out-of-plane contributions are also present but, for the sake of simplicity, are not shown.

proportional to the applied electric field (say along the $x$ direction), as shown in Figs. 1(a) and 1(b), there will be more occupied states with spin up-along $y$-than with spin down. In the case of short-range disorder, the total in-plane polarization can be estimated to be proportional to the density of states multiplied by the shift in momentum, $s_{y}$ $\sim N \delta p \sim N|e| \mathcal{E} \tau$. Since in the present situation we are dealing with the two Fermi surfaces corresponding to the two helicity bands $\epsilon_{ \pm}=p^{2} / 2 m \pm \alpha p$, obtained from the Hamiltonian (1), one expects $s_{y} \sim\left(N_{+}-N_{-}\right) \delta p$, where, for the Rashba interaction, one has $N_{ \pm}=N_{0}\left(1 \mp \alpha / v_{F}\right), N_{0}=m / 2 \pi$. Explicit calculations agree with this simple picture and lead to the result due to Edelstein, ${ }^{15} s_{y}=-N_{0} \alpha|e| \mathcal{E} \tau$. When long-range disorder is considered, a reasonable guess would be to substitute for $\tau$ the transport time $\tau_{t r}$

$$
\tau \rightarrow \tau_{t r}, \quad \frac{1}{\tau_{t r}}=\int d \theta W(\theta)[1-\cos (\theta)],
$$

with $W(\theta)$ being the angle-dependent scattering probability, so that $s_{y}=-N_{0} \alpha|e| \mathcal{E} \tau_{t r}$. This was proposed in Ref. 29; however, the picture is too simplistic, and therefore the guess is wrong. As discussed in Ref. 21, the proper $s_{y}$ polarization is given by $s_{y}=-N_{0} \alpha|e| \mathcal{E} \tau_{E}$, with

$$
\tau \rightarrow \tau_{E}, \quad \frac{1}{\tau_{E}}=\int d \theta W(\theta)[1-\cos (2 \theta)] .
$$

This particular time $\tau_{E}$, where " $E$ " stands for Edelstein, arises from the asymmetric shift of the two Fermi surfaces, as depicted in Fig. 1(c), due to different transport times in the two bands. It shows that contributions from both forward $(\theta=0)$ and backward $(\theta=\pi)$ scatterings are suppressed. The next step is to consider what happens when magnetic impurities are included. Relying once again on the simple picture of the shifted Fermi surface, one could argue that these have a rather small impact on the spin polarization since the spinflip scattering time usually makes a small contribution to the total transport time. However, even when this is the case, magnetic disorder does not simply modify the total transport time but has an additional nontrivial effect. In its presence the spins do not align themselves along the internal $\mathbf{b}$ field since they acquire nonvanishing components in the plane orthogonal to it [see Fig. 1(d)]. It is these components that give rise to a finite spin Hall conductivity. In this respect, magnetic disorder has an effect similar to that of an in-plane magnetic field: it affects the spin-quantization axis and tilts the spins out of their expected stationary direction. We now make these arguments quantitative.

The starting point is the Eilenberger equation, ${ }^{22}$ which we write explicitly for a homogeneous Rashba 2DEG in linear response to a constant and homogeneous applied electric field

$$
\begin{aligned}
\partial_{t} g^{K}= & \mathbf{v}_{F} \cdot \mathcal{E}|e| \partial_{\epsilon} g_{e q}^{K}-\frac{1}{2}\left\{\frac{1}{p_{F}} \partial_{\varphi} \mathbf{b} \cdot \boldsymbol{\sigma}, \mathbf{e}_{\varphi} \cdot \mathcal{E}|e| \partial_{\epsilon} g_{e q}^{K}\right\} \\
& +i\left[\mathbf{b} \cdot \boldsymbol{\sigma}, g^{K}\right]-i[\check{\check{\Sigma}}, \check{g}]^{K} .
\end{aligned}
$$

The quasiclassical Green function $\left[\check{g} \equiv \check{g}_{t_{1} t_{2}}(\hat{\mathbf{p}} ; \mathbf{x})\right]$ is defined as $\left(\xi=p^{2} / 2 m-\mu\right)$

$$
\check{g}=\frac{i}{\pi} \int \mathrm{d} \xi \check{G}_{t_{1} t_{2}}(\mathbf{p}, \mathbf{x}), \quad \check{G}=\left(\begin{array}{cc}
G^{R} & G^{K} \\
0 & G^{A}
\end{array}\right),
$$

where $\check{G}_{t_{1} t_{2}}(\mathbf{p}, \mathbf{x})$ is the Wigner representation of the Green function, which has a matrix structure in both Keldysh (denoted by the check symbol) and spin space. Equation (8) is the equation of motion for the Keldysh component- the one related to physical observables-identified by the superscript " $K$," which will be from now on implicitly assumed and thus dropped. Moreover, $g_{\text {eq }}^{K}=\tanh (\epsilon / 2 T)\left(g_{\text {eq }}^{R}-g_{\text {eq }}^{A}\right)$, where $g_{e q}^{R}=-g_{e q}^{A}=1-\partial_{\xi} \mathbf{b} \cdot \boldsymbol{\sigma}$, indicates the equilibrium-no electric field-function. ${ }^{22}$ All objects are evaluated at the Fermi surface in the absence of spin-orbit coupling while $\varphi$ is the angle defined by the momentum, $\mathbf{p}=p(\cos \varphi, \sin \varphi)$, and $\mathbf{e}_{\varphi}$ $=(-\sin \varphi, \cos \varphi)$. From Eqs. (2) and (3) one obtains the selfenergy contributions

$$
\check{\Sigma}_{\mathrm{nm}}(\mathbf{p})=n_{\mathrm{nm}} \sum_{\mathbf{p}^{\prime}}\left|U\left(\mathbf{p}-\mathbf{p}^{\prime}\right)\right|^{2} \check{G}\left(\mathbf{p}^{\prime}\right),
$$

and

$$
\check{\check{\Sigma}}_{\mathrm{m}}=n_{\mathrm{m}} \frac{B^{2}}{3} \sum_{l=1}^{3} \sum_{\mathbf{p}} \sigma_{l} \check{G}(\mathbf{p}) \sigma_{l},
$$

where $n_{\mathrm{nm}}$ and $n_{\mathrm{m}}$ denote the concentrations of nonmagnetic and magnetic impurities, respectively. In order to consider long-range nonmagnetic disorder, we first expand the nonmagnetic scattering kernel in spherical harmonics of the scattering angle, and neglect its dependence on the modulus of $\mathbf{p}$ and $\mathbf{p}^{\prime}$ 


$$
\begin{aligned}
n_{\mathrm{nm}}|U|^{2}= & \frac{1}{2 \pi N_{0} \tau}\left[1+2 K_{1} \cos \left(\varphi-\varphi^{\prime}\right)\right. \\
& \left.+2 K_{2} \cos \left(2 \varphi-2 \varphi^{\prime}\right)+\ldots\right] \\
\equiv & \frac{1}{2 \pi N_{0} \tau}\left[1+K\left(\varphi-\varphi^{\prime}\right)\right],
\end{aligned}
$$

with $\tau$ the nonmagnetic contribution to the elastic lifetime. Then we write the magnetic scattering kernel in terms of the spin-flip time $\tau_{s f}$,

$$
n_{\mathrm{m}} B^{2}=\frac{1}{2 \pi N_{0} \tau_{s f}} .
$$

The complete disorder self-energy can then be written, separating its $s$-wave and higher harmonics contributions,

$$
\check{\Sigma}=\check{\Sigma}_{\mathrm{m}}+\check{\Sigma}_{\mathrm{nm}}^{1}+\check{\Sigma}_{\mathrm{nm}}^{2}=-\frac{i}{6 \tau_{s f}} \sum_{l=1}^{3} \sigma_{l}\langle\check{g}\rangle \sigma_{l}-\frac{i}{2 \tau}\langle\check{g}\rangle-\frac{i}{2 \tau}\langle K \check{g}\rangle,
$$

where $\langle\ldots\rangle \equiv \int \mathrm{d} \varphi / 2 \pi \ldots$.

The connection between $\check{g}$ and the physical observables is made by integrating over the energy $\epsilon$, which is the Fourier conjugate variable of the time difference $t_{1}-t_{2}$. For instance, the spin density is given by the angular average of the Keldysh component, ${ }^{30}$

$$
\mathbf{S}=\mathbf{s}^{e q}-\frac{N_{0}}{8} \int \mathrm{d} \epsilon\langle\operatorname{Tr}(\boldsymbol{\sigma} g)\rangle .
$$

In order to solve Eq. (8), it is convenient to turn it into matrix form, writing $g$ as a four-vector

$$
g=g_{0} \sigma_{0}+\mathbf{g} \cdot \boldsymbol{\sigma}, \quad\left(g_{\mu}\right)=\left(g_{0}, \mathbf{g}\right) .
$$

Rather than using the standard $\left(\sigma_{x}, \sigma_{y}, \sigma_{z}\right)$ basis, we choose to rotate to $\left(\sigma_{\|}, \sigma_{\perp}, \sigma_{z}\right)$, the subscripts $\|$ and $\perp$, indicating, respectively, the directions parallel and perpendicular to the internal field b. Defining the rotation matrix $\mathbf{R}(\varphi)$ by

$$
\left(\begin{array}{c}
\sigma_{0} \\
\sigma_{x} \\
\sigma_{y} \\
\sigma_{z}
\end{array}\right)=\left(\begin{array}{cccc}
1 & 0 & 0 & 0 \\
0 & \sin \varphi & \cos \varphi & 0 \\
0 & -\cos \varphi & \sin \varphi & 0 \\
0 & 0 & 0 & 1
\end{array}\right)\left(\begin{array}{c}
\sigma_{0} \\
\sigma_{\|} \\
\sigma_{\perp} \\
\sigma_{z}
\end{array}\right)
$$

one has

$$
\begin{aligned}
& g_{\mu}^{\prime}=\sum_{\mu^{\prime}=0}^{3} \mathbf{R}_{\mu \mu^{\prime}}^{-1}(\varphi) g_{\mu^{\prime}}, \quad\left(g_{\mu}^{\prime}\right)=\left(g_{0}, g_{\|}, g_{\perp}, g_{z}\right), \\
& \mathbf{K}_{\mu \nu}\left(\varphi, \varphi^{\prime}\right)=\sum_{\mu^{\prime}=0}^{3} \mathbf{R}_{\mu \mu^{\prime}}^{-1}(\varphi) K\left(\varphi-\varphi^{\prime}\right) \mathbf{R}_{\mu^{\prime} \nu}\left(\varphi^{\prime}\right) .
\end{aligned}
$$

Expanding in harmonics, we also drop the four-vector indices

$$
\mathbf{K}\left(\varphi, \varphi^{\prime}\right)=\mathbf{K}^{(a)}+\cos \left(\varphi-\varphi^{\prime}\right) \mathbf{K}^{(b)}+\sin \left(\varphi-\varphi^{\prime}\right) \mathbf{K}^{(c)}+\ldots
$$

In the above we have defined

$$
\mathbf{K}^{(a)}=\left(\begin{array}{cccc}
0 & 0 & 0 & 0 \\
0 & K_{1} & 0 & 0 \\
0 & 0 & K_{1} & 0 \\
0 & 0 & 0 & 0
\end{array}\right), \quad \mathbf{K}^{(b)}=\left(\begin{array}{cccc}
2 K_{1} & 0 & 0 & 0 \\
0 & K_{2} & 0 & 0 \\
0 & 0 & K_{2} & 0 \\
0 & 0 & 0 & 2 K_{1}
\end{array}\right),
$$

and

$$
\mathbf{K}^{(c)}=\left(\begin{array}{cccc}
0 & 0 & 0 & 0 \\
0 & 0 & -K_{2} & 0 \\
0 & K_{2} & 0 & 0 \\
0 & 0 & 0 & 0
\end{array}\right)
$$

For the purpose of calculating polarizations and spin currents, the higher harmonics play no role and are thus ignored.

By using $g_{e q}^{R}=-g_{e q}^{A}=1-\partial_{\xi} \mathbf{b} \cdot \boldsymbol{\sigma}$ and performing a rotation to the new spin basis, one can write Eq. (8) as

$$
\partial_{t} g^{\prime}=\frac{1}{\tau^{*}}\left[-\mathbf{M} g^{\prime}+\left(\mathbf{N}_{0}+\mathbf{N}_{1}\right)\left\langle g^{\prime}\right\rangle+\left(\mathbf{N}_{2}+\mathbf{N}_{3}\right)\left\langle\mathbf{K} g^{\prime}\right\rangle\right]+S_{\mathcal{E}} .
$$

The matrices appearing in Eq. (23) read

$$
\mathbf{M}=\left(\begin{array}{ccccc}
1 & -\frac{\tau^{*}}{\tau} \frac{\alpha}{v_{F}} K_{1} & 0 & 0 \\
-\frac{\tau^{*}}{\tau} \frac{\alpha}{v_{F}} K_{1} & 1 & 0 & 0 \\
0 & 0 & 1 & 2 \alpha p_{F} \tau^{*} \\
0 & & 0 & -2 \alpha p_{F} \tau^{*} & 1
\end{array}\right),
$$

where $\tau^{*}$ is the elastic quasiparticle lifetime, defined as 


$$
\frac{1}{\tau^{*}} \equiv \frac{1}{\tau}+\frac{1}{\tau_{s f}}
$$

which we now use for convenience of notation but will be later incorporated into the proper transport time. Finally, $S_{\mathcal{E}}$ is the source term due to the electric field. We take this to be along the $x$ direction so that

$$
S_{\mathcal{E}} \equiv|e| v_{F} \mathcal{E} \partial_{\epsilon}[2 \tanh (\epsilon / 2 T)]\left(\begin{array}{c}
\cos \varphi \\
-\cos \varphi \frac{\alpha}{v_{F}} \\
-\sin \varphi \frac{\alpha}{v_{F}} \\
0
\end{array}\right) .
$$

Solving for the $s_{z}$ spin current flowing along $y$, we obtain

$$
\begin{aligned}
& j_{s_{z}}^{y}=-\frac{N_{0}}{4} \int \mathrm{d} \epsilon v_{F}\left\langle\hat{\mathbf{p}}_{y} g_{z}\right\rangle \\
& =-\frac{N_{0}}{4} \int \mathrm{d} \epsilon\left[-\frac{\frac{4}{3 \tau_{s f}}-i \omega}{2 m \alpha}\right]\left(\left\langle\hat{\mathbf{p}}_{y} g_{\perp}\right\rangle-\left\langle\hat{\mathbf{p}}_{x} g_{\|}\right\rangle\right) \\
& =-\frac{N_{0}}{4} \int \mathrm{d} \epsilon\left[-\frac{\frac{4}{3 \tau_{s f}}-i \omega}{2 m \alpha}\right]\left\langle g_{y}\right\rangle \\
& =\left[-\frac{\frac{4}{3 \tau_{s f}}-i \omega}{2 m \alpha}\right] s_{y} \text {, }
\end{aligned}
$$

i.e., the continuity equation result [Eq. (4)] under homogeneous conditions. In the third line we have used Eq. (17) to set $\left\langle g_{y}\right\rangle=\left\langle\hat{\mathbf{p}}_{y} g_{\perp}\right\rangle-\left\langle\hat{\mathbf{p}}_{x} g_{\|}\right\rangle$. Similarly, one obtains the complete expression for the frequency dependent $s_{y}$ spin polarization

$$
\begin{aligned}
s_{y}= & -N_{0} \alpha|e| \mathcal{E} 2\left(\alpha p_{F}\right)^{2}\left[\left(\frac{1}{\tau_{t r}}-i \omega\right)\left(\frac{1}{\tau_{E}}-i \omega\right)\left(\frac{4}{3 \tau_{s f}}-i \omega\right)\right. \\
& \left.+2\left(\alpha p_{F}\right)^{2}\left(\frac{1}{\tau_{E}}+\frac{4}{3 \tau_{s f}}-2 i \omega\right)\right]^{-1} .
\end{aligned}
$$

Besides $1 / \tau_{s f}$, there appear in the above two other different time scales,

$$
\frac{1}{\tau_{t r}} \equiv \frac{1}{\tau}\left(1-K_{1}\right)+\frac{1}{\tau_{s f}}, \quad \frac{1}{\tau_{E}} \equiv \frac{1}{\tau}\left(1-K_{2}\right)+\frac{1}{\tau_{s f}} .
$$

The first, $\tau_{t r}$, is the total transport time. The second, $\tau_{E}$, is the generalization of the characteristic time related to the $s_{y}$ spin polarization introduced in (7). By using Eq. (31) in Eq. (30), one obtains the expression for the frequency dependent spin Hall conductivity

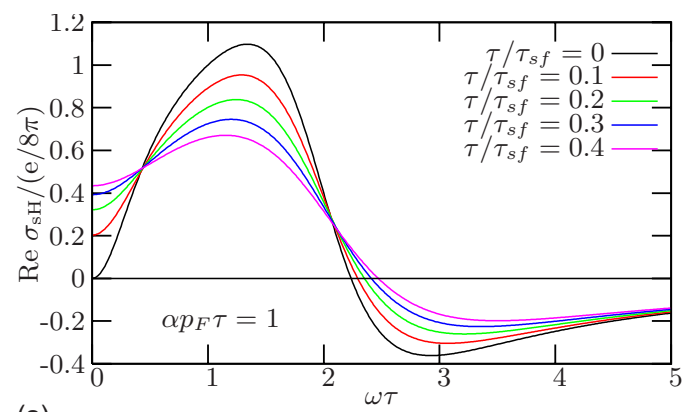

(a)

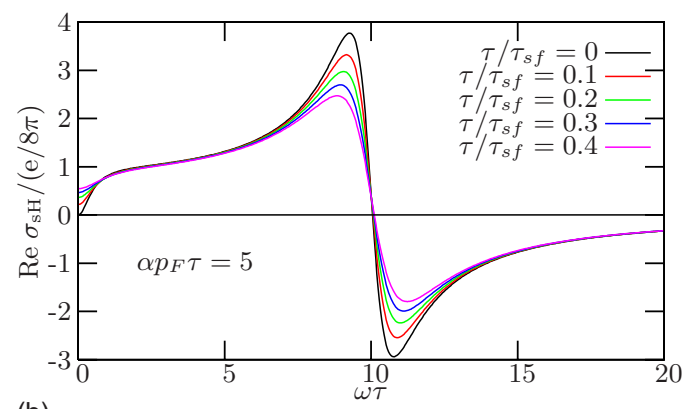

(b)

FIG. 2. (Color online) Real part of the frequency dependent spin Hall conductivity in units of the universal value $|e| / 8 \pi$ for (a) $\alpha p_{F} \tau=1$ and (b) $\alpha p_{F} \tau=5$. The different curves correspond to different values of the ratio $\tau / \tau_{s f}=0,0.1,0.2,0.3,0.4$ (from top to bottom at the maximum of $\operatorname{Re} \sigma_{s H}$ ).

$$
\begin{aligned}
\sigma_{s H}(\omega)= & \frac{|e|}{4 \pi}\left(\frac{4}{3 \tau_{s f}}-i \omega\right) 2\left(\alpha p_{F}\right)^{2}\left[\left(\frac{1}{\tau_{t r}}-i \omega\right)\left(\frac{1}{\tau_{E}}-i \omega\right)\right. \\
& \left.\times\left(\frac{4}{3 \tau_{s f}}-i \omega\right)+2\left(\alpha p_{F}\right)^{2}\left(\frac{1}{\tau_{E}}+\frac{4}{3 \tau_{s f}}-2 i \omega\right)\right]^{-1} .
\end{aligned}
$$

Its real part is displayed in Fig. 2 for different values of the disorder parameter $\alpha p_{F} \tau$. In the limit $\omega \rightarrow 0$, the magnitude of the spin Hall conductivity depends on the value of $\alpha p_{F} \tau$ as well as on the ratio $\tau / \tau_{s f}$. In the absence of magnetic impurities one has the known result $\sigma_{s H}=0$. As spin-flip scattering grows, the conductivity reaches values of the order of the "universal" $|e| / 8 \pi$. This was noted already in Ref. 12, where however, as pointed out in the beginning, angle-dependent scattering was not considered. Large values of $\alpha p_{F} \tau$ can be achieved both in III-V and II-VI semiconducting materials. Doping the latter with Mn allows controlling of the spin-flip time $\tau_{s f}$ while only weakly affecting the electron mobility ${ }^{31-33}$ even though it is not perfectly clear whether these can appropriately be described in terms of the linear Rashba model. ${ }^{34}$ Additionally, for certain frequencies one can see crossing points $[\omega \tau \approx 0.5$ and $\omega \tau \approx 2$ in Fig. $2(\mathrm{a})]$ at which magnetic disorder has no effect on the spin Hall response. Such points are well defined only when $\alpha p_{F} \tau \approx 1$. For clean $\left(\alpha p_{F} \tau \gg 1\right)$ or dirty $\left(\alpha p_{F} \tau \ll 1\right)$ samples, the different curves cross each other over a progressively wider range of frequencies.

Finally, in the diffusive regime, $\omega \tau_{t r} \ll 1, \alpha p_{F} \tau_{t r} \ll 1$, and 
assuming $\tau_{t r} / \tau_{s f} \ll 1, \tau_{E} / \tau_{s f} \ll 1$, one obtains the following spin-diffusion equations:

$$
\begin{aligned}
& \partial_{t} s_{x}=-\left(\frac{1}{\tau_{s}}+\frac{4}{3 \tau_{s f}}\right) s_{x}, \\
& \partial_{t} s_{y}=-\left(\frac{1}{\tau_{s}}+\frac{4}{3 \tau_{s f}}\right) s_{y}-\alpha N_{0}|e| \mathcal{E} \frac{\tau_{E}}{\tau_{s}}, \\
& \partial_{t} s_{z}=-\left(\frac{2}{\tau_{s}}+\frac{4}{3 \tau_{s f}}\right) s_{z},
\end{aligned}
$$

where $\left(2 \alpha p_{F} \tau_{t r}\right)^{2} / 2 \tau_{t r} \equiv 1 / \tau_{s}$ is the D'yakonov-Perel spinrelaxation rate, tied to Rashba spin-orbit coupling. From Eq. (34) the sensitivity of the in-plane spin polarization on spinflip scattering is apparent: in the stationary limit the source (proportional to $\mathcal{E}$ ) is balanced by the spin relaxation. Spinflip scattering leaves the source unchanged, whereas it enhances the relaxation rate so that in the end $s_{y}$ is reduced.

In conclusion, we studied the combined effect of longrange and magnetic disorders on voltage induced spin polarizations and the related spin Hall currents in a Rashba 2DEG. We investigated homogeneous but nonstatic conditions from the dirty $\left(\alpha p_{F} \tau \ll 1\right)$ to the clean $\left(\alpha p_{F} \tau \gg 1\right)$ regime. Care is required when treating long-range disorder because of the two-band structure of the problem while magnetic impurities, even in low concentrations, play a nontrivial role beyond that of a simple redefinition of the time scales.

This work was supported by the Deutsche Forschungsgemeinschaft through SFB 484 and SPP 1285, and by CNISM under Progetti Innesco 2006.
${ }^{1}$ M. I. D'yakonov and V. I. Perel, Phys. Lett. 35A, 459 (1971).

${ }^{2}$ J. E. Hirsch, Phys. Rev. Lett. 83, 1834 (1999).

${ }^{3}$ S. Murakami, N. Nagaosa, and S.-C. Zhang, Science 301, 1348 (2003).

${ }^{4}$ J. Sinova, D. Culcer, Q. Niu, N. A. Sinitsyn, T. Jungwirth, and A. H. MacDonald, Phys. Rev. Lett. 92, 126603 (2004).

${ }^{5}$ J. I. Inoue, G. E. W. Bauer, and L. W. Molenkamp, Phys. Rev. B 70, 041303(R) (2004).

${ }^{6}$ E. G. Mishchenko, A. V. Shytov, and B. I. Halperin, Phys. Rev. Lett. 93, 226602 (2004).

${ }^{7}$ R. Raimondi and P. Schwab, Phys. Rev. B 71, 033311 (2005).

${ }^{8}$ A. Khaetskii, Phys. Rev. Lett. 96, 056602 (2006).

${ }^{9}$ E. I. Rashba, Phys. Rev. B 70, 201309(R) (2004).

${ }^{10}$ O. V. Dimitrova, Phys. Rev. B 71, 245327 (2005).

${ }^{11}$ O. Chalaev and D. Loss, Phys. Rev. B 71, 245318 (2005).

${ }^{12}$ J. I. Inoue, T. Kato, Y. Ishikawa, H. Itoh, G. E. W. Bauer, and L. W. Molenkamp, Phys. Rev. Lett. 97, 046604 (2006).

${ }^{13}$ C. Gorini, P. Schwab, M. Dzierzawa, and R. Raimondi, Physica E (Amsterdam) 40, 1078 (2008).

${ }^{14}$ P. Wang, Y.-Q. Li, and X. Zhao, Phys. Rev. B 75, 075326 (2007).

${ }^{15}$ V. M. Edelstein, Solid State Commun. 73, 233 (1990).

${ }^{16}$ A. G. Aronov and Y. B. Lyanda-Geller, JETP Lett. 50, 431 (1989).

${ }^{17}$ Y. K. Kato, R. C. Myers, A. C. Gossard, and D. D. Awschalom, Phys. Rev. Lett. 93, 176601 (2004).

${ }^{18}$ V. Sih, R. C. Myers, Y. K. Kato, W. H. Lau, A. C. Gossard, and D. D. Awschalom, Nat. Phys. 1, 31 (2005).

${ }^{19}$ C. L. Yang, H. T. He, Lu Ding, L. J. Cui, Y. P. Zeng, J. N. Wang, and W. K. Ge, Phys. Rev. Lett. 96, 186605 (2006).

${ }^{20}$ N. P. Stern, S. Ghosh, G. Xiang, M. Zhu, N. Samarth, and D. D. Awschalom, Phys. Rev. Lett. 97, 126603 (2006).

${ }^{21}$ M. Milletarì, R. Raimondi, and P. Schwab, Europhys. Lett. 82,
67005 (2008).

${ }^{22}$ R. Raimondi, C. Gorini, P. Schwab, and M. Dzierzawa, Phys. Rev. B 74, 035340 (2006).

${ }^{23}$ W.-K. Tse and S. Das Sarma, Phys. Rev. B 74, 245309 (2006).

${ }^{24}$ M. Trushin and J. Schliemann, Phys. Rev. B 75, 155323 (2007).

${ }^{25}$ C.-X. Liu, B. Zhou, S.-Q. Shen, and B.-F. Zhu, Phys. Rev. B 77, 125345 (2008).

${ }^{26} \mathrm{Had}$ we chosen the linear Dresselhaus field, the whole calculation would have gone through without substantial difference. More in general, this holds for any internal field $\mathbf{b}$, where the direction of $\mathbf{b}$ does not depend on the modulus of $\mathbf{p}$.

${ }^{27}$ Several suggestions for the definition of the spin current exist in the literature. We choose a nonconserved spin current $\mathbf{j}_{s_{i}}$ $=1 / 4\left\{\sigma_{i}, \mathbf{v}\right\}, i=x, y, z, \mathbf{v}=\nabla_{\mathbf{p}} H$, which is consistent with the $S U(2)$ covariant formulation, see, e.g., P. Q. Jin, Y. Q. Li, and F. C. Zhang, J. Phys. A 39, 7115 (2006).

${ }^{28}$ D. Culcer and R. Winkler, Phys. Rev. Lett. 99, 226601 (2007).

${ }^{29}$ H. A. Engel, E. I. Rashba, and B. I. Halperin, Phys. Rev. Lett. 98, 036602 (2007).

${ }^{30}$ Within the quasiclassical formalism, $g$ describes the dynamical part only, to which the equilibrium part has to be added.

${ }^{31}$ Y. S. Gui, C. R. Becker, J. Liu, V. Daumer, V. Hock, H. Buhmann, and L. W. Molenkamp, Europhys. Lett. 65, 393 (2004).

${ }^{32}$ V. Daumer, I. Golombek, M. Gbordzoe, E. G. Novik, V. Hock, C. R. Becker, H. Buhmann, and L. W. Molenkamp, Appl. Phys. Lett. 83, 1376 (2003).

${ }^{33}$ S. A. Crooker, D. A. Tulchinsky, J. Levy, D. D. Awschalom, R. Garcia, and N. Samarth, Phys. Rev. Lett. 75, 505 (1995).

${ }^{34}$ More precisely, the Rashba Hamiltonian is appropriate for narrow quantum wells (width $\lesssim 6 \mathrm{~nm}$ ) but most likely not for wider structures, in which the so-called inverted-band structure manifests itself. 